\title{
Evaluación según modernización del Estado en la gestión municipal,
}

2020

\author{
Freddy Seminario Arévalo \\ Seminario_arevalo@ hotmail.com \\ Escuela de posgrado \\ Universidad César Vallejo \\ ORCID: 0000-0001-9900-333X
}

José Manuel Delgado Bardales

jmdelgadob@ucvvirtual.edu.pe

Escuela de posgrado

Universidad César Vallejo

ORCID:0000-0001-6574-2759

Scopus autor ID: 24070333700

Código Renacyt: P0050554

\section{RESUMEN}

El estudio tuvo como objetivo caracterizar la evaluación según la modernización del Estado en la gestión municipal, 2020, la investigación es básica de diseño descriptivo con revisión sistemática, se realizó como procedimiento de recolección de datos una tabla de registro de datos y la fuente fue revisión bibliográfica de artículos de investigaciones tanto nacionales como internacionales que tuvieron como tema principal la evaluación según la modernización del Estado en la gestión municipal; se revisaron (10) artículos más relevantes. En conclusión la evaluación según la modernización del Estado en la gestión municipal, que el 100\% de los estudios relacionados a la evaluación según modernización del Estado reconocen las bondades y beneficios en el contexto de la gestión púbica, debido a que la evaluación demanda compromiso institucional, políticas, condiciones y consecuencias; y se puede establecer que la evaluación tiene un impacto en la gestión lógicamente distinta en diferentes países del mundo, Por otro lado, el $80 \%$ concluye que la evaluación es importante para la gestión pública en el marco de la modernización del Estado, porque permite determinar las condiciones, funciones, toma de decisiones e intervención oportuna para el cumplimiento de metas y objetivos institucionales.

Palabras clave: Evaluación, modernización del estado, gestión municipal 


\title{
Evaluation according to modernization of the State in municipal management, 2020
}

\begin{abstract}
:
The study aimed to characterize the evaluation according to the modernization of the State in municipal management, 2020, the research is basic of descriptive design with systematic review, a data record table was carried out as a data collection procedure and the source was review bibliography of both national and international research articles whose main theme was evaluation according to the modernization of the State in municipal management; (10) most relevant articles were reviewed. In conclusion, the evaluation according to the modernization of the State in municipal management, that $100 \%$ of the studies related to the evaluation according to the modernization of the State recognize the benefits and benefits in the context of public management, because the evaluation demands institutional commitment, policies, conditions and consequences; and it can be established that the evaluation has a logically different impact on management in different countries of the world. On the other hand, $80 \%$ conclude that the evaluation is important for public management within the framework of the modernization of the State, because it allows determining the conditions, functions, decision-making and timely intervention for the fulfillment of institutional goals and objectives.
\end{abstract}

Keywords: Evaluation, modernization of the state, municipal management

Artículo recibido: 03 nov. 2020

Aceptado para publicación: 07 dic. 2020

Correspondencia seminario arevalo@ hotmail.com.

Conflictos de Interés: Ninguna que declarar 


\section{INTRODUCCIÓN}

En la actualidad en el mundo, uno de los muchos retos de gran magnitud que asumen los gobiernos de bajos recursos es el desarrollo y consolidación de un sistema de evaluación que permita conformar una política sistemática de valoración de los resultados de la acción pública, por lo menos en lo que toca a las dependencias y programas. El también llamado sistema de evaluación del desempeño (SED), es producto de la adopción de la gestión por resultados $(\mathrm{GpR})$ y su concomitante modelo de presupuesto basado en resultados $(\mathrm{PbR})$ que responden a la modernización del Estado para generar un gasto y valor público óptimo (González, Hernández, 2010).

Sin embargo, a finales de los ochenta se produjo en América Latina un intenso proceso de reformas del Estado, caracterizado por cuatro elementos centrales: democratización, descentralización, disminución/recuperación del papel del Estado y elevada influencia ejercida por estados Unidos. Desde entonces, se han introducido elecciones municipales en todos los países de América Latina, y de gobernadores provinciales o departamentales. Además, el gasto de los gobiernos subnacionales paso de un $10 \%$ del gasto público total a comienzos de los años ochenta hasta un $35 \%$ a finales de la década de 2000, sin embargo, este periodo se caracterizó por la crisis de deuda externa. Las causas principales del cambio se pueden resumir en la llegada al poder de partidos de izquierda en América Latina, causada por la elevada pobreza y desigualdad que generaron el estancamiento económico y la eliminación de políticas sociales, la caída del bloque soviético, que disminuyó las tensiones entre las dos visiones extremas de la intervención pública (Estado mínimo y máximo) (Bandeira, 2015).

En este sentido, es importante destacar la influencia que han ejercido en las reformas del Estado de América Latina hasta hace pocos años la Agencia de los Estados Unidos para el Desarrollo Internacional (USAID), el Banco Mundial, el Banco Interamericano de Desarrollo y el Fondo Monetario Internacional, quienes han canalizado (a través de préstamos) sumas importantes de dinero hacia los Gobiernos de América Latina y han tratado por este medio de influir en el tipo de reformas que debían llevar a cabo para ello. El grado actual de profundización democrática y capacidad del Estado de cada país lo podemos determinar a partir de los indicadores de libertades políticas, eficacia gubernamental y Estado de derecho recopilados por Kaufman, Kraay y Mastruzzi. A partir de la media de estos indicadores, podemos clasificar los países de América Latina 
en cuatro grupos, en función del grado de desarrollo de sus Estados: Alto: Chile, Uruguay y Costa Rica. Medio: Brasil, Panamá, México, Colombia, Argentina, Perú y El Salvador. Bajo: República Dominicana, Bolivia, Paraguay, Honduras, Ecuador, Guatemala, Nicaragua y Cuba. Muy bajo: Venezuela y Haití (Bandeira, 2015).

Por lo tanto, el Estado busca el mejoramiento constante, que requiere de la intervención de múltiples actores: los políticos, los funcionarios públicos y los propios usuarios de los servicios, para que su funcionamiento impacte en todo el país, principalmente en los sectores menos favorecidos, ya que por su naturaleza, la ayuda que ellos reciben es proporcionalmente mayor, de esta forma, permite el desarrollo de las personas, contribuyendo o limitando el despliegue de sus capacidades y competencias con el fin de alcanzar sus objetivos como individuos, lo que en sí lleva implícita la visión de la sociedad que se desea construir. En todo caso, las distintas tendencias en la gestión pública que incluye la gestión municipal busca impactar el Estado y desde su institucionalidad producir el desarrollo económico, político y social que la población demanda, especialmente en la provisión de bienes públicos, para lo cual debe incorporarse con fuerza los procesos tecnológicos, denominado gobierno electrónico, así como la profesionalización de su recurso fundamental que son los funcionarios alcaldes y equipos de gestión municipal (Pasco, 2015).

En el Perú, el proceso de modernización y adopción de la innovación es joven en el sector público. La Organización para la Cooperación y el Desarrollo Económico - OCDE (2017), realizó una evaluación de la situación de la política de innovación en el país, la cual consistió en una revisión del funcionamiento y el desempeño del sistema de innovación, y de los factores que lo afectan, con énfasis en el papel del gobierno. Este estudio formuló recomendaciones de política en ciencia, tecnología e innovación con el fin de hacerlas sostenibles. No obstante, el crecimiento económico se mantiene rezagado en materia de ingreso per cápita e inversión en capital humano y conocimiento, en comparación con los demás países latinoamericanos. La evaluación también indica una preferencia por el uso de tecnología importada .en desmedro del desarrollo de capacidades propias de innovación. Sin embargo, el desarrollo del país no solo depende del crecimiento económico, sino de la generación de capacidades de innovación en el campo tecnológico. 
Sin embargo, el país cuenta con una política pública aprobada por la Presidencia del Consejo de Ministros (PCM), mediante el Decreto Supremo 027-2013-PCM (2013), centrada en la innovación tecnológica, con el respaldo de programas financiados por préstamos de organismos multilaterales; pero no se cumple a pesar que en su mayoría va dirigidos a promocionar la innovación empresarial. Únicamente la Ley Orgánica de Gobiernos Regionales 27867 (2002), incluye un tema de innovación, aunque restringido a la materia educativa, cuya ejecución no ha sido objeto de evaluación; motivo por el cual no existe medición ni evidencia empírica acerca de su aplicación. La Ley Orgánica de Municipalidades 27972 (2003), no contiene ninguna disposición referida a la innovación; es decir, no la establece como exigencia en su misión, funciones y competencias.

Además, la gestión municipal del gobierno local de Morales debería involucrar el progreso y el conjunto de actividades, estrategias y técnicas apropiadas destinadas a conocer y verificar el desarrollo interno, que satisface a la ciudadanía como un gobierno serio, estratega, eficiente, eficaz, responsable y moderno para cumplir con las expectativas y resolver las demandas y requisitos de los ciudadanos que responda a los requerimientos del Estado acorde a lo tecnológico. De otro lado, el municipio, por su naturaleza, concentran su capacidad de gestión directa y autónoma de los recursos sin mejorar o desarrollar nuevos servicios o políticas públicas en los sectores vivienda, salud, educación, ambiente y turismo, entre otros, a escala provincial, distrital, de centro poblado, metropolitano, fronterizo o rural. Algunos autores sostienen que las municipalidades son las instancias de gobierno democrático más cercanas a la población e, incluso, en lugares alejados de nuestro país solo cuentan con una gestión que afecta las condiciones y la calidad de vida de los vecinos de su comunidad. Sin embargo, en la actualidad el municipio no está totalmente comprometido con el desarrollo local para generen valor público a partir de la búsqueda de soluciones creativas a los problemas y las necesidades de los ciudadanos, que se concretan en cambios o reformas institucionales. En ese contexto el trabajo de investigación propone un modelo de evaluación acorde a los lineamientos de modernización del Estado para elevar la gestión municipal en el distrito de Morales.

\section{ESTRATEGIAS METODOLÓGICAS O MATERIALES Y MÉTODOS}

El tipo de investigación es básica de diseño descriptivo con revisión sistemática, las revisiones sistemáticas son investigaciones científicas en las cuales la unidad de análisis 
son los estudios originales primarios, constituyen una herramienta esencial para sintetizar la información científica disponible, incrementar la validez de las conclusiones de estudios individuales e identificar áreas de incertidumbre donde sea necesario realizar investigación (Hernández, 2016, p.217). Es descriptiva, porque se recogió información relacionada a la evaluación según la modernización del Estado en la gestión municipal (Tamayo y Tamayo, 2009, p. 54). Por lo mencionado, se revisó información científica y se visualizó como solucionar una necesidad en la organización de la entidad pública, para mejorar la evaluación según la modernización del Estado en la gestión municipal y otras deficiencias existentes.

La recolección de datos se realizó a través de una tabla de registro de datos y la fuente fue revisión bibliográfica de artículos de investigaciones tanto nacionales como internacionales que tuvieron como tema principal la evaluación según la modernización del Estado en la gestión municipal; de todos los artículos que se encontraron, se incluyeron los estudios (10) más importantes según nivel de evidencia y se excluyeron los menos relevantes. Se estableció la búsqueda siempre y cuando se tuvo acceso al texto completo del artículo científico.

Se desarrolló a través del análisis de la revisión sistemática evaluando cada uno de los artículos para una comparación de los puntos o características en las cuales concuerda y los puntos en los que existe discrepancia entre artículos nacionales e internacionales. Además, de acuerdo a criterios técnicos pre establecidos, se realizó una evaluación crítica e intensiva de cada artículo, a partir de ello, se determinó la calidad de la evidencia y la fuerza de recomendación para cada artículo 


\section{RESULTADOS Y DISCUSIÓN}

Tabla 1: Estudios revisados sobre la evaluación según la modernización del Estado en la gestión municipal

\section{DATOS DE LA PUBLICACIÓN}

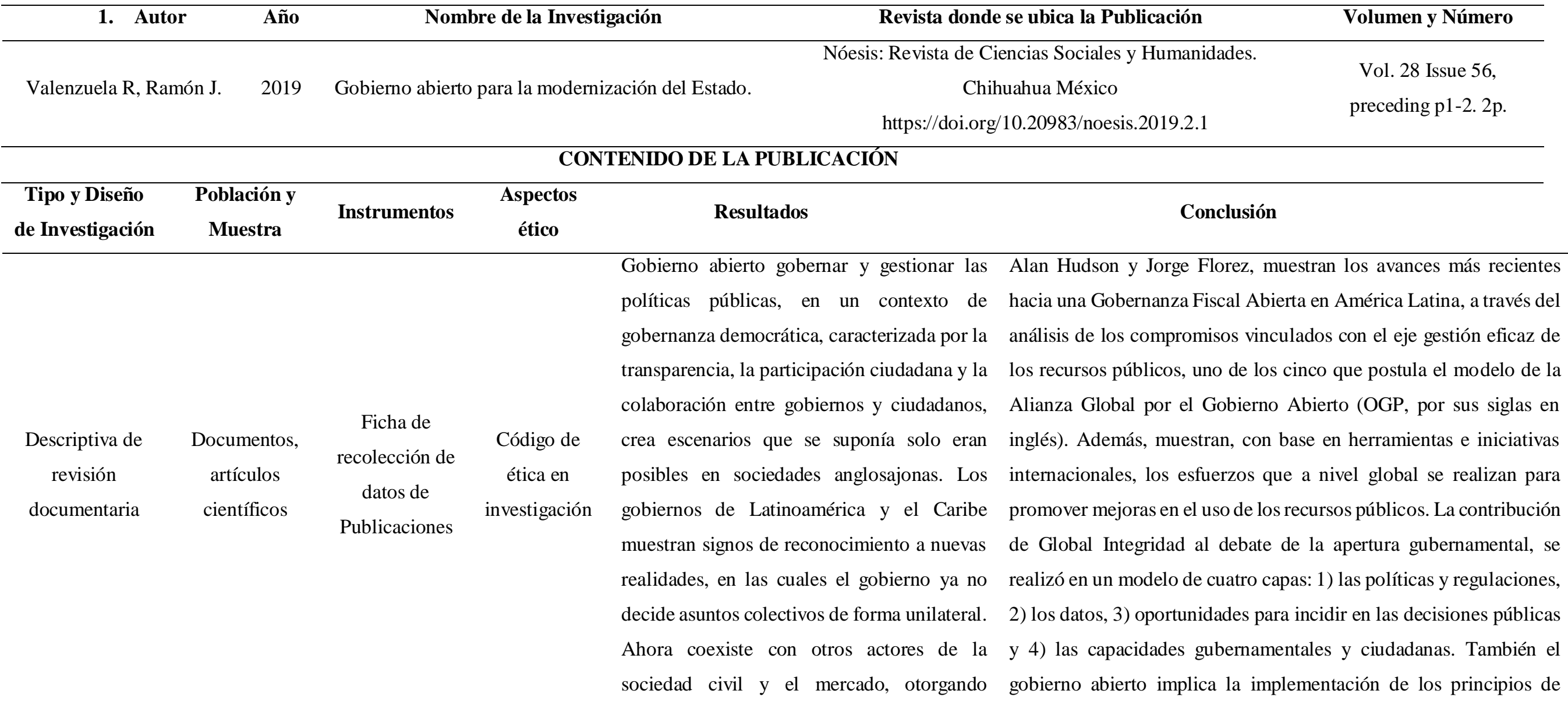


validez a nuevos mecanismos y estrategias para decidir los asuntos públicos de forma conjunta. transparencia, participación ciudadana y rendición de cuentas, y su alcance tiene un valor práctico y transformador, en las relaciones entre ciudadanía y gobierno.

\begin{tabular}{|c|c|c|c|c|c|}
\hline 2. Autor & Año & \multicolumn{2}{|c|}{ Nombre de la Investigación } & Revista donde se ubica la Publicación & \multirow[b]{2}{*}{ Vol. 20 Issue 68, p11-34. 24p. } \\
\hline $\begin{array}{c}\text { Cardona D, Cortés J, } \\
\text { Ujueta S. }\end{array}$ & 2015 & $\begin{array}{l}\text { Gobierno Electrón } \\
\text { marco normativo y } \\
\text { índices estratégicos. }\end{array}$ & $\begin{array}{l}\text { ico en Colombia: } \\
\text { evaluación de tres }\end{array}$ & $\begin{array}{l}\text { Utopia y Praxis Latinoamericana, Maracaibo, Zulia } \\
\text { Venezuela } \\
\text { http://search.ebscohost.com/login.aspx?direct=true\&db= } \\
\text { fua\&AN=108747746\&lang=es\&site=ehost-live }\end{array}$ & \\
\hline \multicolumn{6}{|c|}{ CONTENIDO DE LA PUBLICACIÓN } \\
\hline $\begin{array}{l}\text { Tipo y Diseño de } \\
\text { Investigación }\end{array}$ & $\begin{array}{c}\text { Población y } \\
\text { Muestra }\end{array}$ & Instrumentos & Aspectos ético & Resultados & Conclusión \\
\hline $\begin{array}{l}\text { Descriptiva de revisión } \\
\text { de base de datos }\end{array}$ & $\begin{array}{c}\text { Bases de } \\
\text { datos }\end{array}$ & $\begin{array}{l}\text { análisis de } \\
\text { tendencias de } \\
\text { estos índices, se } \\
\text { empleó } \\
\text { regresión lineal, } \\
\text { posiciones } \\
\text { relativas y } \\
\text { cuartiles }\end{array}$ & $\begin{array}{l}\text { Código de ética } \\
\text { en investigación }\end{array}$ & $\begin{array}{ll}\text { Los resultados permiten evidenciar una } & \text { El Gobierno } \\
\text { constante evolución del marco normativo. } & \text { principalmente } \\
\text { La evaluación sobre los índices no es } & \text { consignadas en } \\
\text { concluyente. Si bien Colombia tiene } & \text { la infraestructu } \\
\text { positivas calificaciones en stock de Capital } & \text { de formación y } \\
\text { Humano y alta presencia Web por parte del } & \text { uso y apropiac } \\
\text { Gobierno, es esencial avanzar en } & \text { ampliar la cob } \\
\text { infraestructura tecnológica y trabajar } & \text { provisión de se } \\
\text { alrededor de la percepción de corrupción por } & \text { eficiente, tran } \\
\text { parte de la ciudadanía } & \text { ciudadana en la }\end{array}$ & $\begin{array}{l}\text { cesita fortalecer aspectos de entorno, } \\
\text { onsolidar el cumplimiento de las metas } \\
\text { marco normativo; ampliar y fortalecer } \\
\text { tecnológica en conjunto con programas } \\
\text { apacitación del Capital Humano para el } \\
\text { n de las TIC. De esta manera, puede } \\
\text { tura de las estrategias de GE para la } \\
\text { icios públicos, y de este modo, ser más } \\
\text { arente y permitir la participación } \\
\text { ma de decisiones públicas. }\end{array}$ \\
\hline
\end{tabular}

Ciencia Latina Revista Científica Multidisciplinar, Ciudad de México, México.

ISSN 2707-2207 / ISSN 2707-2215 (en línea), julio-diciembre, 2020, Volumen 4, Número 2. https://doi.org/10.37811/cl_rcm.v4i2.131 p. 921 


\begin{tabular}{|c|c|c|c|c|c|c|c|}
\hline 3. Autor & & Año & \multicolumn{2}{|c|}{ Nombre de la Investigación } & \multicolumn{2}{|c|}{ Revista donde se ubica la Publicación } & \multirow{2}{*}{$\begin{array}{c}\begin{array}{c}\text { Volumen y } \\
\text { Número }\end{array} \\
\text { Vol. } 15 \text { Issue 2, } \\
\text { p183-199. 17p. }\end{array}$} \\
\hline Alonso L. & & 2016 & \multicolumn{2}{|c|}{$\begin{array}{l}\text { Racionalización, reforma y } \\
\text { modernización administrativa: estado } \\
\text { del proceso en Galicia. }\end{array}$} & $\begin{array}{r}\text { Revista de Investigac } \\
\text { ttp://search.ebscohost } \\
590\end{array}$ & $\begin{array}{l}\text { iones Políticas y Sociológicas (RIPS). Santiago } \\
\text { de Compostela España. } \\
\text { com/login.aspx?direct=true\&db=fua\&AN=125 } \\
\text { 396\&lang=es\&site=ehost-live }\end{array}$ & \\
\hline \multicolumn{8}{|c|}{ CONTENIDO DE LA PUBLICACIÓN } \\
\hline $\begin{array}{l}\text { Tipo y Diseño de } \\
\text { Investigación }\end{array}$ & $\begin{array}{c}\text { Población y } \\
\text { Muestra }\end{array}$ & Inst & umentos & $\begin{array}{l}\text { Aspectos } \\
\text { ético }\end{array}$ & Resultados & Conclusión & \\
\hline Descriptivo & Documentos & & $\begin{array}{l}\text { cha de } \\
\text { istro de } \\
\text { datos }\end{array}$ & $\begin{array}{c}\text { Código de } \\
\text { ética en } \\
\text { investigación }\end{array}$ & $\begin{array}{l}\text { En las últimas décadas, las reformas } \\
\text { hechas con el objetivo de reformar y } \\
\text { consolidar la situación financiera de las } \\
\text { comunidades autónomas basándose en } \\
\text { transferir a éstas servicios y } \\
\text { competencias sin perfeccionar por } \\
\text { completo el modelo de financiamiento, } \\
\text { de modo que se pueda crear una } \\
\text { vinculación efectiva real entre gastos e } \\
\text { ingresos públicos de las comunidades } \\
\text { autónomas }\end{array}$ & $\begin{array}{l}\text { La necesidad de adaptar la administración públic } \\
\text { de la ciudadanía y la situación de profunda rece } \\
\text { en los últimos años evidenció las grandes defi } \\
\text { administrativo solapado y sobredimensionado, } \\
\text { gestión del gasto público. Las cuentas públicas } \\
\text { caracterizado por un gasto elevado y una caída } \\
\text { que debía solventarse a través de una reforma } \\
\text { puesta en marcha de proyectos de racionaliz } \\
\text { administrativa en los distintos niveles de gobie } \\
\text { búsqueda de un servicio público eficiente y } \\
\text { electrónicos y digitales. Este proceso de moder } \\
\text { adecuado sistema de incentivos para todos los ag }\end{array}$ & $\begin{array}{l}\text { a las demandas reales } \\
\text { ión económica vivida } \\
\text { iencias de un aparato } \\
\text { lo que perjudica a la } \\
\text { eflejaban un escenario } \\
\text { rástica de los ingresos } \\
\text { e corte estructural: la } \\
\text { ción y simplificación } \\
\text { lo combinados con la } \\
\text { l uso de los medios } \\
\text { ización precisa de un } \\
\text { ntes involucrados. }\end{array}$ \\
\hline
\end{tabular}

Ciencia Latina Revista Científica Multidisciplinar, Ciudad de México, México. ISSN 2707-2207 / ISSN 2707-2215 (en línea), julio-diciembre, 2020, Volumen 4, Número 2. https://doi.org/10.37811/cl_rcm.v4i2.131 p. 922 


\begin{tabular}{|c|c|c|c|c|c|}
\hline 4. Autor & Año & Nombre de la Investigación & \multicolumn{2}{|c|}{ Revista donde se ubica la Publicación } & \multirow{2}{*}{$\begin{array}{l}\text { Volumen y Número } \\
\text { Vol. } 23 \text { Issue 4, p222- } \\
\text { 233. 12p. } 1 \text { Diagram. }\end{array}$} \\
\hline García R, Sotolongo M. & 2019 & $\begin{array}{l}\text { La evaluación del impacto de la } \\
\text { sistemas de pago por resultados } \\
\text { AGROFAR. }\end{array}$ & $\begin{array}{r}\text { Folletos Gerencia } \\
\text { http://search.ebscohost. } \\
\& \mathrm{db}=\text { fua\&AN=141691 }\end{array}$ & $\begin{array}{l}\text { les. El Vedado Cuba. } \\
\text { com/login.aspx?direct=true } \\
\text { 216\&lang=es\&site=ehost- } \\
\text { live }\end{array}$ & \\
\hline \multicolumn{6}{|c|}{ CONTENIDO DE LA PUBLICACIÓN } \\
\hline Tipo y Diseño de Investigación & $\begin{array}{c}\text { Población y } \\
\text { Muestra }\end{array}$ & Aspectos ético & Resultados & \multicolumn{2}{|c|}{ Conclusión } \\
\hline Descriptiva & $\begin{array}{l}\text { Documentos, } \\
\text { gestores y } \\
\text { expertos }\end{array}$ & $\begin{array}{l}\text { Revisión de } \\
\text { documentos, } \\
\text { encuestas, } \\
\text { tormenta de } \\
\text { ideas, trabajo Código de ética } \\
\text { en grupos, en investigación } \\
\text { observación } \\
\text { directa y } \\
\text { criterio de } \\
\text { expertos. }\end{array}$ & $\begin{array}{l}\text { Se obtuvo una tendencia } \\
\text { positiva en el comportamiento } \\
\text { de los indicadores que miden } \\
\text { los dos niveles de evaluación, } \\
\text { con categoría de bien en el } \\
\text { índice de impacto }\end{array}$ & $\begin{array}{l}\text { Las principales propuestas } \\
\text { en: el perfeccionamiento d } \\
\text { de las capacidades in } \\
\text { incremento de las acciones } \\
\text { las exigencias de cada camf } \\
\text { logística oportuna con pro } \\
\text { eventuales, la moderniza } \\
\text { existente (riego, maquinari } \\
\text { de } 5 \text { años }\end{array}$ & $\begin{array}{l}\text { e mejoras se concentran } \\
\text { la planificación a partir } \\
\text { aladas existentes, el } \\
\text { le capacitación acorde a } \\
\text { ña, logro de una gestión } \\
\text { eedores permanentes y } \\
\text { ión de la tecnología } \\
\text { e industria) en un plazo }\end{array}$ \\
\hline
\end{tabular}




\begin{tabular}{|c|c|c|c|c|c|}
\hline 5. Autor & Año & \multicolumn{2}{|c|}{ Nombre de la Investigación } & Revista donde se ubica la Publicación & Volumen y Número \\
\hline Armesto A. & 2017 & $\begin{array}{l}\text { Evaluación del d } \\
\text { local y aproba } \\
\text { México. }\end{array}$ & $\begin{array}{l}\text { esempeño del gobie } \\
\text { ción presidencial }\end{array}$ & $\begin{array}{l}\text { Frontera Norte. Tijuana México } \\
\text { http://search.ebscohost.com/login.aspx?direct=true } \\
\& \mathrm{db}=\mathrm{a} 9 \mathrm{~h} \& \mathrm{AN}=122029805 \& \text { lang=es\&site=ehost- } \\
\text { live (Acceso: } 7 \text { de junio de 2020). }\end{array}$ & $\begin{array}{l}\text { Vol. } 29 \text { Issue } 57, \mathrm{p} 155- \\
\text { 176. } 22 \mathrm{p} \text {. }\end{array}$ \\
\hline & & & ONTENIDO DE & LA PUBLICACIÓN & \\
\hline Tipo y Diseño de Investigación & $\begin{array}{c}\text { Población y } \\
\text { Muestra }\end{array}$ & Instrumentos & Aspectos ético & Resultados & sión \\
\hline Descriptivo correlacional & $\begin{array}{l}\text { Funcionarios } \\
\text { de los } \\
\text { gobiernos } \\
\text { locales y } \\
\text { usuarios de } \\
\text { los servicios } \\
\text { públicos }\end{array}$ & $\begin{array}{c}\text { Encuestas- } \\
\text { cuestionario, } \\
\text { entrevistas - } \\
\text { guía de } \\
\text { entrevista, ficha } \\
\text { de registro de } \\
\text { datos }\end{array}$ & $\begin{array}{l}\text { Código de ética } \\
\text { en investigación }\end{array}$ & $\begin{array}{lrll}\text { Los resultados } & \text { de } & \text { las } & \text { Investigaciones anteriores } \\
\text { regresiones } & \text { logísticas } & \text { impacto de la economía y d } \\
\text { ordinales y } & \text { regresiones } & \text { aprobación presidencial. L } \\
\text { aparentemente } & \text { no } & \text { de voto retrospectivo se ba } \\
\text { relacionadas indican que la } & \text { los ciudadanos saben quién } \\
\text { satisfacción con los servicios } & \text { las políticas recompensan o } \\
\text { prestados por los gobiernos } & \text { partidos en consecuencia. } \\
\text { locales se } \quad \text { asocia } & \text { sobre la claridad de la } \\
\text { positivamente con } \quad \text { la } & \text { sistemas multinivel, debe } \\
\text { aprobación presidencial } & \text { efectos indirectos del créc } \\
& & \text { todos los niveles de gobier } \\
& & \text { en qué responsabilidades p } \\
& & \text { los diferentes niveles de } \\
& \text { ciudadanos experimentan }\end{array}$ & $\begin{array}{l}\text { an identificado un claro } \\
\text { sempeño de la política de } \\
\text { s propuestas del modelo } \\
\text { an en el supuesto de que } \\
\text { es responsable por el cual } \\
\text { castigan a los políticos o } \\
\text { asándose en la literatura } \\
\text { responsabilidad, en los } \\
\text { amos esperar encontrar } \\
\text { ito para las políticas en } \\
\text { lo. En sistemas políticos } \\
\text { líticas se comparten entre } \\
\text { gobierno y donde los } \\
\text { lificultades para asignar }\end{array}$ \\
\hline
\end{tabular}


correctamente el crédito y la culpa, los índices de aprobación de los políticos en determinados niveles de gobierno se ven afectados por la política

rendimiento en otros niveles

6. Autor

García-García D.
Año

Nombre de la Investigación

Evaluación de políticas públicas

2017 juveniles en tres municipios del Estado de México (2013-2015)
Revista donde se ubica la Publicación

Revista Latinoamericana de Ciencias Sociales,

Niñez y Juventud, 15(1), 403-418.

Manizales - Caldas Colombia

https://doi.org/10.11600/1692715x.151252801201
Volumen y Número

Vol. 15 Issue 1, p403-

418. $16 \mathrm{p}$.

\section{CONTENIDO DE LA PUBLICACIÓN}

\begin{tabular}{|c|c|c|c|c|c|}
\hline $\begin{array}{l}\text { Tipo y Diseño de } \\
\text { Investigación }\end{array}$ & $\begin{array}{c}\text { Población y } \\
\text { Muestra }\end{array}$ & Instrumentos & Aspectos ético & Resultados & Conclusión \\
\hline $\begin{array}{l}\text { Aplicada, descriptiva } \\
\text { enfoque de análisis } \\
\text { comparado }\end{array}$ & $\begin{array}{c}\text { Bibliografía, } \\
\text { documentos, } \\
\text { programas } \\
\text { digitales }\end{array}$ & $\begin{array}{l}\text { herramientas de } \\
\text { la investigación } \\
\text { documental y } \\
\text { de campo }\end{array}$ & $\begin{array}{l}\text { Código de ética } \\
\text { en investigación }\end{array}$ & $\begin{array}{l}\text { No se encontraron políticas públicas } \\
\text { municipales en materia de juventud. } \\
\text { En su lugar, se ejecutaron acciones } \\
\text { desarticuladas, desorganizadas, } \\
\text { eventuales, que pueden llamarse } \\
\text { "políticas de improvisación" en } \\
\text { materia de juventud, las cuales } \\
\text { evidenciaron una ausencia completa } \\
\text { de planeación. }\end{array}$ & $\begin{array}{l}\text { Las "políticas públicas" municipales en materia de juventud } \\
\text { siguieron teniendo en la práctica una visión adulto céntrica, } \\
\text { ven a los jóvenes como sujetos de riesgo a quienes hay que } \\
\text { "atender" bajo supuestos normativos y medicalizantes. Se } \\
\text { trabaja sin contar con diagnósticos sobre la condición juvenil. } \\
\text { Los presupuestos destinados a los "espacios" } \\
\text { institucionalizados para adelantar políticas públicas para } \\
\text { atender las poblaciones juveniles (Consejos de Juventud, } \\
\text { Institutos de Juventud, Casas de Juventud) son limitados, y no }\end{array}$ \\
\hline
\end{tabular}

Ciencia Latina Revista Científica Multidisciplinar, Ciudad de México, México.

ISSN 2707-2207 / ISSN 2707-2215 (en línea), julio-diciembre, 2020, Volumen 4, Número 2. https://doi.org/10.37811/cl_rcm.v4i2.131 p. 925 
Los municipios estudiados no es posible conocer cuánto presupuesto se les destinó, ya que aprobaron ejercicios ciudadanos ni siquiera las propias autoridades conocen el dato exacto. sobre transparencia y rendición de Hay pocas evidencias que se impulsó el triángulo mágico de cuentas en el tema juvenil elaboración, diseño, implementación y evaluación de las "políticas públicas" municipales sobre juventud, y no hay participación de organizaciones civiles y juveniles ni de instituciones académicas en las políticas públicas.

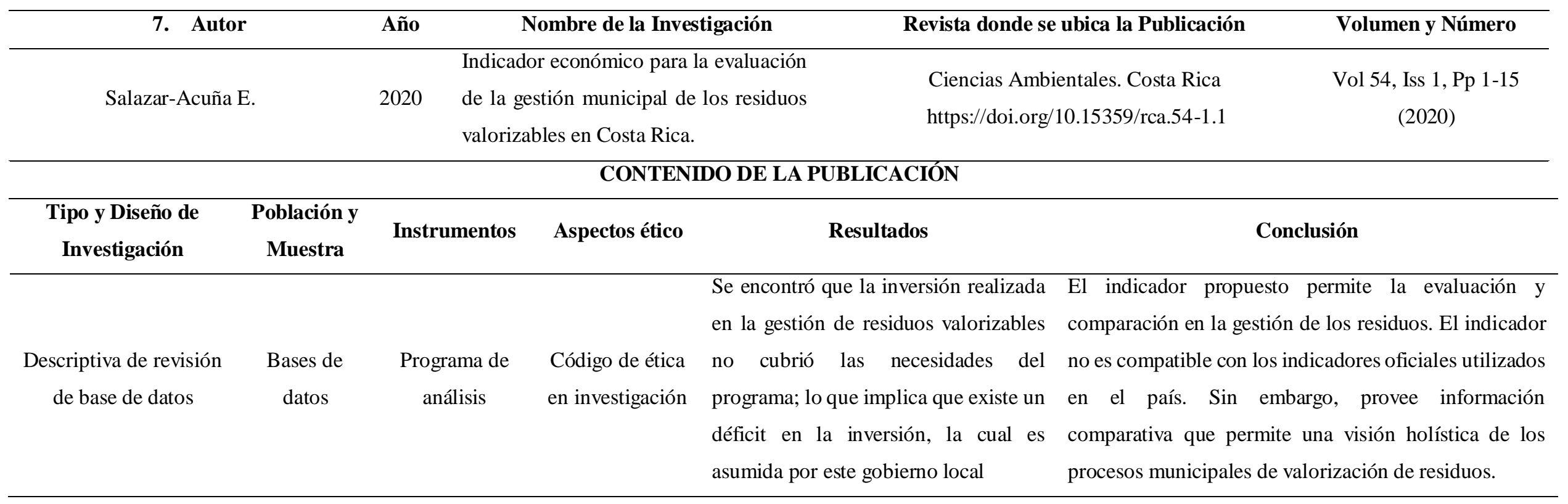

Ciencia Latina Revista Científica Multidisciplinar, Ciudad de México, México. ISSN 2707-2207 / ISSN 2707-2215 (en línea), julio-diciembre, 2020, Volumen 4, Número 2. https://doi.org/10.37811/cl_rcm.v4i2.131 p. 926 


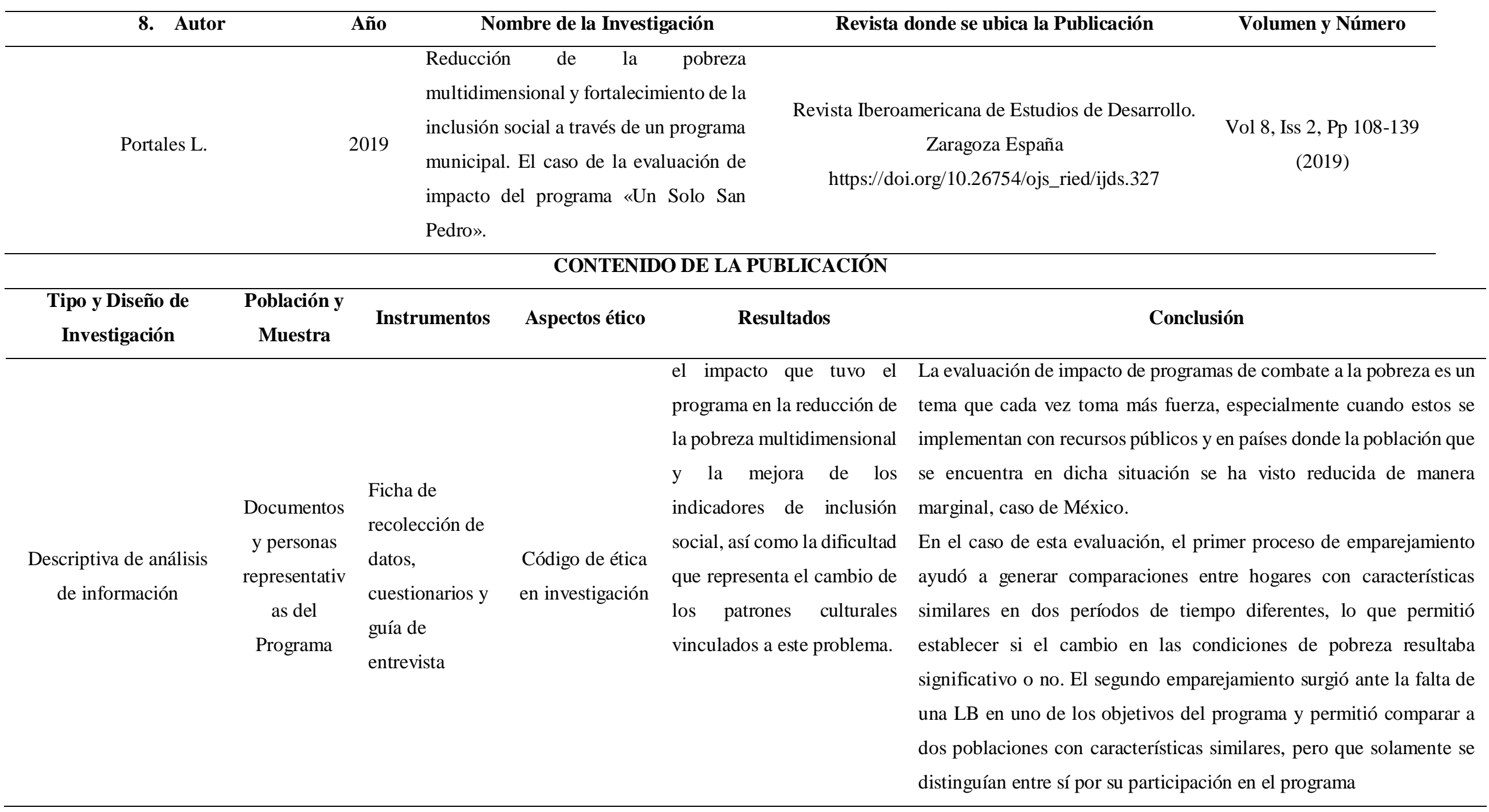




\begin{tabular}{|c|c|c|c|c|}
\hline 9. Autor & Año & Nombre de la Investigación & Revista donde se ubica la Publicación & Volumen y Número \\
\hline Gutiérrez F, Rodríguez S. & 2018 & $\begin{array}{l}\text { Evaluación del Plan de Inversión } \\
\text { Municipal de Siuna Región Autónoma } \\
\text { de la Costa Caribe Norte de Nicaragua, } \\
2016 .\end{array}$ & $\begin{array}{c}\text { Revista Universitaria Del Caribe. Nicaragua. } \\
\text { https://doi.org/10.5377/ruc.v19i2.6469 }\end{array}$ & $\begin{array}{c}\text { Vol 19, Iss 2, Pp 36-46 } \\
\text { (2018) }\end{array}$ \\
\hline
\end{tabular}

\begin{tabular}{|c|c|c|c|c|c|}
\hline \multicolumn{6}{|c|}{ CONTENIDO DE LA PUBLICACIÓN } \\
\hline $\begin{array}{l}\text { Tipo y Diseño de } \\
\text { Investigación }\end{array}$ & $\begin{array}{c}\text { Población y } \\
\text { Muestra }\end{array}$ & Instrumentos & Aspectos ético & Resultados & Conclusión \\
\hline
\end{tabular}

Ciencia Latina Revista Científica Multidisciplinar, Ciudad de México, México. ISSN 2707-2207 / ISSN 2707-2215 (en línea), julio-diciembre, 2020, Volumen 4, Número 2. https://doi.org/10.37811/cl_rcm.v4i2.131 p. 928 


\begin{tabular}{|c|c|c|c|c|}
\hline 10. Autor & Año & Nombre de la Investigación & Revista donde se ubica la Publicación & Volumen y Número \\
\hline $\begin{array}{l}\text { Pérez-López, R., Morales-Sánchez } \\
\text { V., Anguera, M. T., \& Hernández- } \\
\text { Mendo, A. }\end{array}$ & 2015 & $\begin{array}{l}\text { Evaluación de la calidad total } \\
\text { servicios municipales deporti } \\
\text { orientados a la población infat } \\
\text { aportaciones desde el análisis cualita } \\
\text { con ATLAS.ti }\end{array}$ & $\begin{array}{l}\text { Cuadernos de Psicología Del Deporte. Barcelona } \\
\text { España } \\
\text { https://doi.org/10.4321/S1578- } \\
84232015000100014\end{array}$ & $\begin{array}{l}\text { CPD vol.15 n.1 Murcia J } \\
\text { an. } 2015\end{array}$ \\
\hline & & CONTENIDO DE & AA PUBLICACIÓN & \\
\hline Cualitativo & $\begin{array}{l}\text { Directivos, } \\
\text { colaboradore } \\
\text { s y usuarios } \\
\text { (padres y } \\
\text { niños9 }\end{array}$ & $\begin{array}{cc}\text { Guía de } & \\
\text { entrevista, guía } & \text { Código de ética } \\
\text { de grupos } & \text { en investigación } \\
\text { focales } & \end{array}$ & $\begin{array}{lrl}\text { Los resultados } & \text { estimados } & \text { Los/las usuarios/as infant } \\
\text { indican, que } & \text { los/las } & \text { total de los servicios depc } \\
\text { usuarios/as dan una relevancia } & \text { importante a los aspectos } \\
\text { importante a la dimensión de } & \text { aspectos emocionales y de } \\
\text { aspectos } & \text { tangibles, } & \text { funcionales de la calidad d } \\
\text { principalmente al atributo } & \\
\text { referido a la apariencia de las } & \\
\text { instalaciones físicas, material } & \\
\text { y limpieza } & \end{array}$ & $\begin{array}{l}\text { es al evaluar la calidad } \\
\text { tivos dan una relevancia } \\
\text { tangibles, y destacan los } \\
\text { elación afectiva sobre los } \\
1 \text { servicio }\end{array}$ \\
\hline
\end{tabular}




\section{Discusión}

Posterior a la revisión sistemática de los artículos, del 100\%, el 30\% corresponden a España, el 70\% a Latinoamérica y el Caribe, a los países de México, Venezuela, Cuba, Colombia, Costa Rica y Nicaragua. En relación a los tipos y diseños estudios el 100\% son descriptivos con variantes en revisión documentaria, análisis de bases de datos, correlacional aplicada de análisis comparativo cualitativa.

Valenzuela, Ramón, concluyen que evaluar el gobernar y gestionar las políticas públicas, en un contexto de gobernanza democrática, es caracterizada por la transparencia de la participación y la colaboración entre gobierno y ciudadanos para crear escenarios integrales que sólo eran posibles en sociedades anglosajonas, sin embargo, ello responde a la gestión eficaz de los recursos públicos a través del análisis de compromisos.

Cardona y Cortés, establecen que la evaluación del gobierno fortalece aspectos del entorno para consolidar el cumplimiento de metas según marco normativo, ampliar y fortalecer la estructura tecnológica y la capacitación del capital humano con el uso de las TIC.

Alonso, concluye que la evaluación según la modernización del Estado demanda adaptar la administración pública a demandas reales de la sociedad y a la situación de profunda recesión económica de los últimos años, considerando al aparato administrativo solapado y sobredimensionado, lo que perjudica a la gestión del gasto público.

García y Sotolongo, determina en relación a la evaluación se concentra en el perfeccionamiento de la planificación según capacidades instaladas el incremento de capacitaciones acorde a las exigencias que demanda la modernidad, con adecuada gestión logística, acompañada de una modernización de la tecnología existente.

Armesto, concluye que la evaluación responde al compromiso de los ciudadanos que realizan vigilancia del impacto económico y desempeño de la política pública presidencial, por ende, las políticas recompensan o castigan a los políticos representantes del Estado y debe estar presente en todos los niveles de gobierno.

García-García, establece que la evaluación según modernización del Estado responde a evaluar las políticas públicas como programas dirigidos a grupos específicos donde muchas veces se observa acciones desarticuladas, desorganizadas, eventuales a las cuales pueden llamarse políticas de improvisación porque evidencia completa de planeación. 
Por su parte Salazar- Acuña, establece que los indicadores permiten evaluación y comparación en el desempeño institucional, estos deben oficiales en el país para comparar y tener una visión holística de los procesos municipales.

Además, Portales, determina que la evaluación de impacto de programas del Estado combate la pobreza especialmente cuando son implementados con recursos públicos. Asimismo, establece que la evaluación es el primer para generar comparaciones entre diferentes situaciones para establecer el cambio en las condiciones de pobreza.

Gutiérrez y Rodríguez, concluyen que la evaluación se da bajo el contexto de la planificación de inversión municipal en los diferentes niveles de gobierno, el mismo puede ser dimensionado en los ámbitos políticos, sociales, económicos y ambientales. Por ello es importante la pertinencia social y económica del plan de inversiones en el territorio.

Pérez-López, Morales-Sánchez, Agüera y Hernández-Mendo, determina que las evaluaciones a través de los usuarios dan una relevancia importante a la dimensión de aspectos tangibles y los aspectos emocionales para la calidad del servicio

\section{CONCLUSIÓN O CONSIDERACIONES FINALES}

La revisión sistemática de los 10 artículos científicos, sobre la evaluación según la modernización del Estado, corresponde a diferentes bases de datos, y todos ellos corresponden al tipo y diseño de estudios descriptivos en sus diferentes variantes.

Posterior a la revisión sistémica, se concluye que el 100\% de los estudios relacionados a la evaluación según modernización del Estado reconocen las bondades y beneficios en el contexto de la gestión púbica, debido a que la evaluación demanda compromiso institucional, políticas, condiciones y consecuencias; y se puede establecer que la evaluación tiene un impacto en la gestión lógicamente distinta en diferentes países del mundo.

Por otro lado, el $80 \%$ concluye que la evaluación es importante para la gestión pública en el marco de la modernización del Estado, porque permite determinar las condiciones, funciones, toma de decisiones e intervención oportuna para el cumplimiento de metas y objetivos institucionales.

La evaluación también permite determinar las capacidades y competencias desarrolladas en el recurso humano para que tome un camino diferente a favor del desarrollo institucional asegurando la vida sostenible a pesar de la crisis económica de los países, porque hay acciones que no depende de dinero sino del comportamiento de los colaboradores. 
El liderazgo y el trabajo en equipo multidisciplinario y multisectorial es la herramienta de cambio para la mejorar la evaluación en la gestión pública y garantizar un desarrollo sostenible para las nuevas generaciones.

\section{LISTA DE REFERENCIAS}

Alonso L. (2016). Racionalización, reforma y modernización administrativa: estado del proceso en Galicia. Revista de Investigaciones Políticas y Sociológicas (RIPS). Vol. 15 Issue 2, p183-199. 17p. Santiago de Compostela España. Disponible en: ttp://search.ebscohost.com/login.aspx?direct=true \&db=fua\&AN=125590396\&1 ang $=$ es \& site $=$ ehost-live

Arias, R. (2019). Gestión municipal de agua y gestión del presupuesto en la Municipalidad Provincial de Pativilca, 2019. Artículo. Universidad César Vallejo. Lima Perú. (Artículo científico) http://repositorio.ucv.edu.pe/handle/UCV/42701

Armesto A. (2017). Evaluación del desempeño del gobierno local y aprobación presidencial en México. Frontera Norte. Vol. 29 Issue 57, p155-176. 22p.Tijuana México. Disponible en: http://search.ebscohost.com/login.aspx?direct=true \&db=a9h\&AN=122029805 \&lang=es\&site=ehost-live (Acceso: 7 de junio de 2020).

Bandeira P. (2015). Las reformas del Estado en América Latina: Situación actual y retos. Universidad Francisco de Vitoria. Revista Iberoamericana de Estudios de Desarrollo / Iberoamerican Journal of Development Studies Volumen/volume 4, número/issue 1, pp. 36-55. ISSN: 2254-2035. España. file:///C:/Users/LENOVO/Downloads/Dialnet-

LasReformasDelEstadoEnAmericaLatina-5059580.pdf

Cardona D, Cortés J, Ujueta S. (2015). Gobierno Electrónico en Colombia: marco normativo y evaluación de tres índices estratégicos. Utopía y Praxis Latinoamericana, Maracaibo, Zulia Venezuela. Vol. 20 Issue 68, p11-34. 24p. Disponible http://search.ebscohost.com/login.aspx?direct=true $\& d b=$ fua $\& A N=108747746$ \&lang=es\&site=ehost-live

Cornejo, M. (2019). Análisis de la gestión municipal de Pucusana - Lima en el marco de la Modernización del Estado. (Artículo científico). Universidad César Vallejo. Lima 
Perú.

http://repositorio.ucv.edu.pe/bitstream/handle/UCV/27260/Cornejo_SME.pdf?se quence $=1 \&$ isAllowed $=\mathrm{y}$

Fonseca, F; Vásquez, R; Prado, O. (2011). Evaluación de la capacidad de gobierno: reflexiones sobre la experiencia del Premio 'Municipios que Fazem Render Mais' (2010 e 2011). Revista de Administracao Publica-RAP, vol. 47, no. 1, 2013, p. 249+. Gale Academic OneFile, https://link.gale.com/apps/doc/A432273721/AONE?u=univcv\&sid=AONE\&xid $=24 \mathrm{f} 62 \mathrm{~d} 3 \mathrm{~b}$. Accessed 20 May 2020 .

García-García D. (2017). Evaluación de políticas públicas juveniles en tres municipios del Estado de México (2013-2015). Revista Latinoamericana de Ciencias Sociales, Niñez y Juventud, 15(1), 403-418. Vol. 15 Issue 1, p403-418. 16p. Manizales Caldas Colombia. Disponible en: https://doi.org/10.11600/1692715x.1512528012016

García R, Sotolongo M. (2019). La evaluación del impacto de los sistemas de pago por resultados en AGROFAR. Folletos Gerenciales. El Vedado Cuba. Vol. 23 Issue 4, p222-233. 12p. 1 Diagram. Disponible en: http://search.ebscohost.com/login.aspx?direct=true \&db=fua\&AN=141691216\&1 ang $=$ es\& site $=$ ehost-live

González D, Hernández E. (2010). La política de evaluación del Gobierno mexicano: Crítica desde una perspectiva conceptual. Articulo. Revista de Administración Pública. ISSN: 0482-5209 www.inap.org.mx Instituto Nacional de Administración Pública, $\quad$ A.C $\quad$ México. http://www.inap.mx/portal/images/REVISTA_A_P/rap121.pdf

Grandinetti, R. (2014), Diseño de organizaciones públicas locales para la gestión de territorios complejos: hacia una propuesta metodológica. (Tesis Doctoral). Universidad Politécnica de Valencia. España. https://dialnet.unirioja.es/servlet/tesis?codigo $=88682$

Grupo gestor. (2008). Instrumentos para la autoevaluación de la gestión municipal, señales de un bueno gobierno para el desarrollo local. El Salvador. Primera edición. Editado por ISDEM/GTZ/FUNDE. http://www.repo.funde.org/94/ 
Gutiérrez F, Rodríguez S. (2018). Evaluación del Plan de Inversión Municipal de Siuna Región Autónoma de la Costa Caribe Norte de Nicaragua, 2016. Revista Universitaria Del Caribe. Vol 19, Iss 2, Pp 36-46 (2018). Nicaragua. Disponible en: https://doi.org/10.5377/ruc.v19i2.6469

Kroth, Ch; De Meireles, R. (2019). The effects of time on the performance of local government: the "Pact for Health Care" Program. RAP: Revista Brasileira de Administração Pública, [s. 1.], v. 53, n. 6, p. 1138-1160, 2019. DOI 10.1590/0034761220180440x.Disponívelem:

http://search.ebscohost.com/login.aspx?direct=true $\& d b=a 9 h \& A N=141284221 \&$ lang=es\&site=eds-live. Acesso em: 20 maio. 2020.

Miranda-Tortoló, T; Machado-Martínez, H; Lezcano-Fleires, J; Suset-Pérez, A; OropesaCasanova, K; Tirado-García, F. (2019). Aprendizajes en el proceso de gestión del desarrollo local en un municipio matancero. Pastos y Forrajes, 42(1), 73+. $\begin{array}{llll}\text { Matanzas } & \text { Cuba. } & \text { Retrieved }\end{array}$ https:/link.gale.com/apps/doc/A595143423/AONE?u=univcv\&sid=AONE\&xid $=6 \operatorname{ceb} 227 \mathrm{f}$

Organización para la Economía Cooperación y desarrollo - OECD. (2017). La Contratación Pública en el Perú: Reforzando Capacidad y Coordinación. Estudios de la OCDE sobre Gobernanza Pública, OECD Publishing, Paris, https://doi.org/10.1787/9789264281356-es..

Pasco J. (2015). La modernización del Estado en el Perú. Revista estudio de políticas públicas. 2 (noviembre). Universidad de Chile. Lima Perú. file:///C:/Users/LENOVO/Downloads/Dialnet-

LaModernizacionDelEstadoEnElPeru-6067298.pdf

Pérez-López, R., Morales-Sánchez, V., Anguera, M., \& Hernández-Mendo, A. (2015). Evaluación de la calidad total en servicios municipales deportivos orientados a la población infantil: aportaciones desde el análisis cualitativo con ATLAS Ti Cuadernos de Psicología Del Deporte. CPD vol.15 n.1 Murcia Jan. 2015Barcelona España. Disponible en: https://doi.org/10.4321/S1578-84232015000100014

Presidencia del Consejo de Ministros. (2013). Decreto supremo No 027-2013-PCM. Diario oficial El peruano. Lima Perú. http://www.pcm.gob.pe/normaslegales/2013/DS027-2013-PCM.pdf 
Presidencia del Consejo de Ministros. (2002). Ley Orgánica de Gobiernos Regionales 27867.LimaPerú.

https://portal.jne.gob.pe/portal_documentos/files/informacionlegal/Constitucin\% 20y\%20Leyes1/LEY\%20ORGANICA\%20DE\%20GOBIERNOS\%20REGION ALES.pdf

Presidencia del Consejo de Ministros (2003). La Ley Orgánica de Municipalidades 27972. Lima

Perú. http://www2.congreso.gob.pe/sicr/cendocbib/con4_uibd.nsf/BCD316201CA9C DCA05258100005DBE7A/\$FILE/1_2.Compendio-normativo-OT.pdf

Portales L. (2019). Reducción de la pobreza multidimensional y fortalecimiento de la inclusión social a través de un programa municipal. El caso de la evaluación de impacto del programa «Un Solo San Pedro». Revista Iberoamericana de Estudios de Desarrollo. Vol 8, Iss 2, Pp 108-139 (2019). Zaragoza España. Disponible en: https://doi.org/10.26754/ojs_ried/ijds.327

Quispe, J. (2020). Modelo de open government para optimizar la gestión municipal del gobierno local de Jayanca. Artículo. Universidad César Vallejo. Chiclayo Perú. http://repositorio.ucv.edu.pe/bitstream/handle/UCV/42985/Quispe_DLSJL.pdf?s equence $=1 \&$ isAllowed $=\mathrm{y}$

Salazar-Acuña, E. (2020). Indicador económico para la evaluación de la gestión municipal de los residuos valorizables en Costa Rica. Artículo. https://doi.org/10.15359/rca.54-1.1

http://search.ebscohost.com/login.aspx?direct=true $\& d b=e d s b a s \& A N=e d s b a s . E 3$ 501BB1\&lang=es\&site=eds-live. Acesso em: 20 maio. 2020.

Samuel, M; Stanescu, C; Cardozo, M. (2011). Análisis estratégico de la evaluación de la calidad del servicio en el sector público. Compendium, 14(27),39-59. http://search.ebscohost.com/login.aspx ?direct=true $\& d b=$ fua $\& A N=82582198 \& l a$ ng=es\& site $=$ eds-live.

Valenzuela R, Ramón J. (2019). Gobierno abierto para la modernización del Estado. Nóesis: Revista de Ciencias Sociales y Humanidades. Vol. 28 Issue 56, preceding p1-2. 2p. Chihuahua México. Disponible en: https://doi.org/10.20983/noesis.2019.2.1 